\title{
Performance Study of IEEE 802.15.4 for Industrial Maintenance Applications
}

\author{
Nicolas Salles, Nicolas Krommenacker and Vincent Lecuire \\ Centre de Recherche en Automatique de Nancy (CRAN - UMR 7039), \\ Nancy-Université, CNRS \\ Faculté des Sciences et Techniques, BP 239, \\ F-54 506 Vandœuvre-lès-Nancy CEDEX, FRANCE \\ Email: firstname.lastname@cran.uhp-nancy.fr
}

\begin{abstract}
Nowadays, a growing number of enterprises are adopting wireless technologies. However, field communications induce real-time constraints and those networks should at least fulfill those requirements. In this paper, we study IEEE 802.15.4 performances in order to support industrial real-time traffic. This analysis brings out a comparative analysis of IEEE 802.15.4 medium access methods. It provides also some limitations of that standard concerning cycle duration and amount of messages per cycle.
\end{abstract}

\section{INTRODUCTION}

Over the past few years, intensive wireless networks developments have been done and there are more and more used for industrial cases [1]. Wireless networks provide noticeable advantages in terms of mobility and costs reduction. Nevertheless wireless channel is errorprone and packet losses are inevitable due to noise, multipath delay, fading effects. These phenomena emphasize in industrial environment where production machinery, heavy obstructions and metal structures impact radio wave propagation.

Nowadays, a major problem in designing industrial wireless networks is hard real-time and reliability requirements [2]. So wireless technologies are mainly used in monitoring and surveillance applications where a loss of a few in formations will not be a major inconvenience. For instance, wireless networks are used for predictive maintenance, aboard an operating oil tanker in [3] and on a plant floor in [4]. In the European Integrated Project DYNAMITE [5], new low cost and self-powered sensors with wireless connectivity are developed in order to instrument machines for predictive maintenance. So, the process monitoring could be easily achieved by maintenance crews through hand-held mobile device, nearby the machine. Such application requires simple wireless connectivity, relaxed throughput, very low power, short distance and inexpensive. That's why, the IEEE 802.15.4 [6] technology has been adopted among Bluetooth or IEEE 802.11 technologies. But monitoring traffic involves, for a certain part, real-time communications. Consequently, the ability of the IEEE 802.15.4 to support time-constraint traffic must be studied. In this paper, we present a performance analysis of IEEE 802.15.4 to support real-time applications. We consider for comparison each channel access methods provided by 802.15 .4 . The first one is unslotted CSMA/CA (Carrier Sense Multiple Access / Collision Avoidance). Of course, CSMA/CA is not collision-free but it is always possible to develop a deterministic protocol at the upper layers (e.g. master/slave). The second one includes slotted CSMA/CA and GTS (Guaranteed Time Slots) which allow bounded time unidirectional transmissions.

Although several network topologies are possible with 802.15.4 (simple star topology, peer-to-peer topology and cluster tree), only the simple star which corresponds to the needs expressed in the DYNAMITE Project, is considered in this paper. In star topology (see figure 1), the communication is established between end devices and a single central controller, called the coordinator. The coordinator is a Full-Function Device (FFD) and is generally full-powered. In the DYNAMITE scenario, this device is situated nearby the machine. Sensors are the end devices. They are designed as Reduced-Function Device (RFD) for low cost and low complexity purposes. The mobile device operated by the maintenance crew (i.e. PDA) is also considered as an end device.

This paper is organized as follow. In Section II we overview the IEEE 802.15.4 specifications. Section III comments on network configuration. Section IV focuses on performance analysis of the reservation mechanism. Then it is compared to CSMA/CA in Section V. Section VI relates our contribution with previous works on 


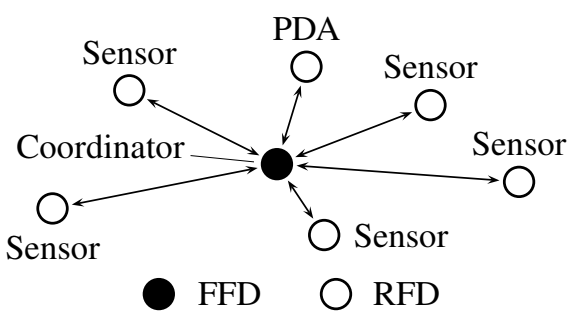

Figure 1. Network architecture

performance analysis of industrial wireless communications. Finally, this paper concludes in Section VII.

\section{IEEE 802.15.4 OVERVIEW}

\section{A. Medium access schemes}

802.15.4 defines two kinds of medium access control namely beacon-enabled and non beacon-enabled. The coordinator decides what mode to use. In non-beacon mode, channel access mechanism is CSMA/CA. Advantages of this mode are scalability and self-organization. However, the non beacon-enabled mode cannot provide any time guarantees to deliver data frames.

In beacon mode, channel time is divided into different periods according to a superframe structure, as shown in figure 2 .

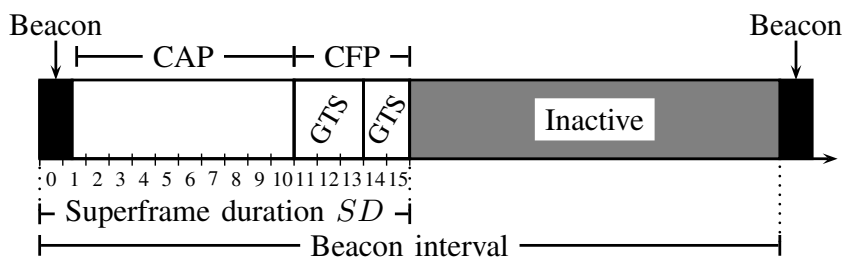

Figure 2. Superframe structure

This superframe consists of an active portion, during which the coordinator interacts with the end devices, and an optional inactive portion, during which all devices may enter a low power mode to reduce power consumption. Each superframe is bounded by network beacons, which are periodically broadcast by the coordinator device to synchronize the attached devices and to describe the structure of the superframe.

The active portion of each superframe consists of 16 equally sized slots and is divided into a Contention Access Period (CAP) and an optional Contention-Free Period (CFP). CAP starts immediately after the beacon. During the CAP, any device wishing to communicate shall compete with other devices using a slotted CSMA/CA mechanism. CFP, if present, is divided into GTS's. A GTS allows communication between a device and the coordinator within a portion of time which is dedicated exclusively to that device. The GTS direction is specified as either transmit or receive. Each device may request one transmit GTS and/or one receive GTS. No more than 7 GTS's can be simultaneously allocated in the superframe.

\section{B. Superframe parameters}

Our study focuses on performances evaluation of such a network. Therefore, we consider the shortest inactive period without considering energy consumption contrary to [7]. Inactive period may be null size. As a result, we don't consider power-saving in the latter. Superframe duration equals beacon interval and is equivalent to cycle duration. Superframe duration is defined by :

$$
S D=a B S F D \times 2^{S O} \text { with } 0 \leq S O \leq 14
$$

$S O$ is an integer value which corresponds to beacon order. This parameter is defined by the network coordinator whereas $a B S F D$ is specified by the standard. $a B S F D$ corresponds to the initial and minimum superframe duration.

Superframe durations are presented figure 3 depending on the network parameters set by the coordinator (channel/frequency, modulation and superframe order).

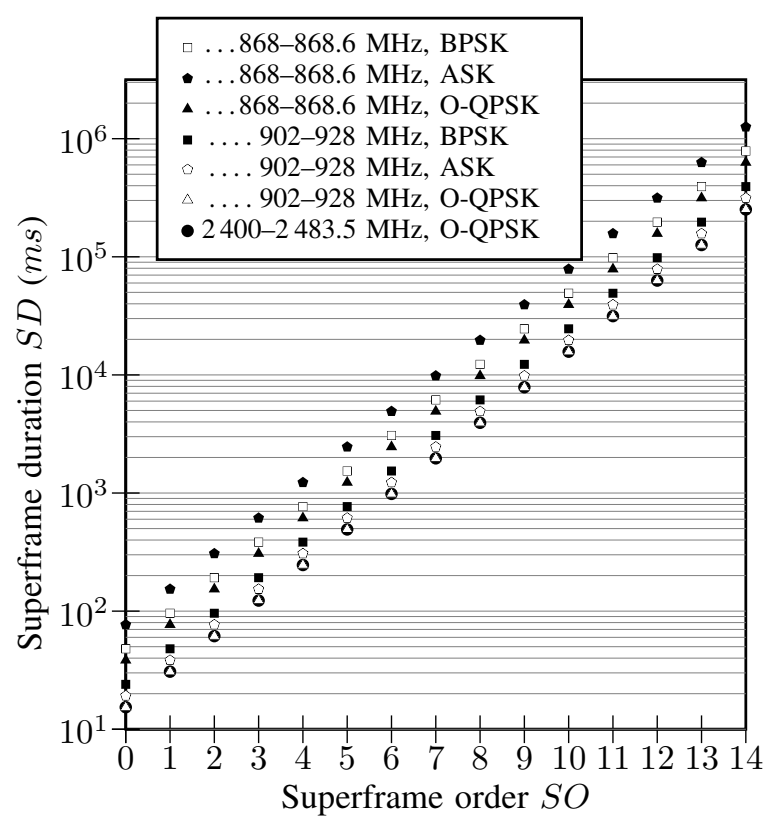

Figure 3. Superframe duration in relation with network parameters

We figure out that most efficient network parameters implies the use of a O-QPSK modulation in association with a frequency in the range either $902-928 \mathrm{MHz}$ or 
2400-2 483.5 MHz. The use of such a configuration results in superframe duration included between $15.36 \mathrm{~ms}$ and $251.6 \mathrm{~s}$. Due to the fact the first range of frequencies is optional for standard specification while the second one is mandatory, we only take care of the use of band $2400-2483.5 \mathrm{MHz}$ in this paper. So the remaining parameter the coordinator has to define, is the superframe order.

\section{NETWORK CONFIGURATION}

In order to evaluate a superframe duration, we have to know how a network is configured. So we need to specify the value of the undefined parameter, superframe order $S O$. More the superframe order is low, more the superframe duration is low and so more the cycle duration is accurate for real-time communications. Choice of superframe order must be as low as possible also it respects constraints concerning standard specification and usage of the network. In the next two subsections, we focus on those two constraints.

\section{A. Specification constraints}

CFP usage implies the respect of the transmission of the beacon frame as well as the minimum CAP duration, aMinCAPLength. Then in order to evaluate the impact of a superframe order value, we must consider the mandatory slots $M_{\text {slots }}$ required by these two transmissions. CAP length is defined by the coordinator and so it is considered as the minimum required by the standard aMinCAPLength. In contrary, beacon frame size, which is application dependent, is considered as its maximum size Beacon $_{\max }=133$ bytes headers included. Thereby, we determine (1), (2) the maximum CFP length in terms of remaining slots $R_{\text {slots }}$ depending on the superframe order (see fig. 4).

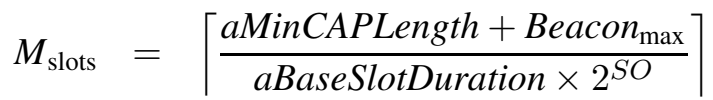

$$
\begin{aligned}
& R_{\text {slots }}=16-M_{\text {slots }}
\end{aligned}
$$

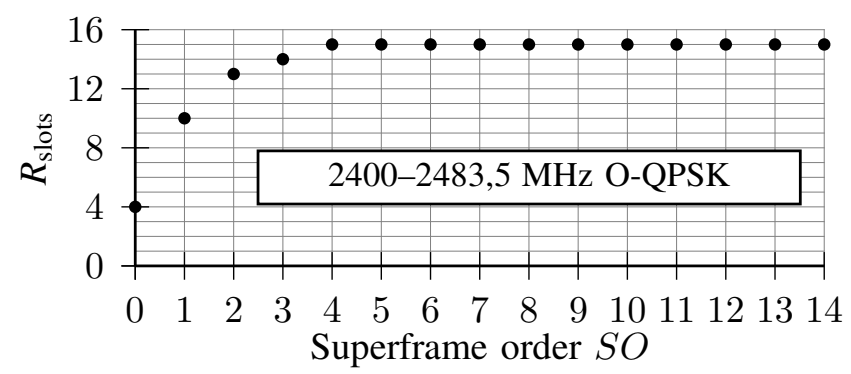

Figure 4. Maximum available slots for CFP
So, choice of a superframe can be checked over the minimum required by the specification. For example, if CFP has to be 5 slots long, it may be impossible to satisfy all requirements with $S O=0$. In such a case, superframe order must be chosen at least equal to 1 .

\section{B. Usage constraints}

In order to evaluate the choice of a superframe order, we must define how many slots are needed for CFP. In that part, we deal with CFP slots requirements depending on data transmission. We consider a unidirectional transmission between two devices of Data bytes in one GTS. That amount of data is considered at the application layer and will be encapsulated by the two IEEE 802.15.4 layers in order to form a network packet. For a communication in a GTS, we consider $P H Y_{\text {headers }}=6$ bytes and $M A C_{\text {headers }}=23$ bytes. $M A C_{\text {headers }}$ is defined considering the maximum value for auxiliary security field as we can't control the use of such a parameter in industrial environments.

For 1 byte of payload data, we send at least 30 bytes over the network including 29 bytes corresponding to encapsulation. In addition, we should also take care of transmission scheme specified by the standard and notably of interframe spaces (IFS) which should be part of GTS. Figure 5 explains that mechanism which should follow every packets send over the network. It depends size of transmitted packets and acknowledgment configuration.

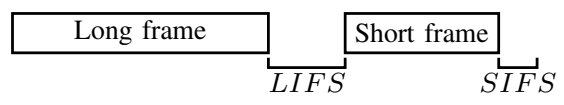

(a) unacknowledged

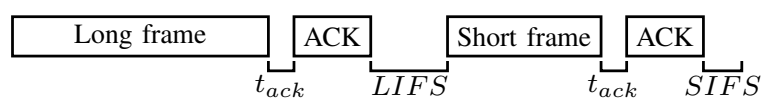

(b) acknowledged

Figure 5. IEEE 802.15.4 interframe spaces

Short frames correspond to packets which length is lower or equal to 24 bytes. Due to the encapsulation $\left(P H Y_{\text {headers }}+M A C_{\text {headers }}=29\right.$ bytes $)$ considered in that paper, we don't develop what concerns short frames and only consider transmission of long frames in both case, without and with acknowledgment. So, we define in (3), the total amount of bytes Data $_{\text {transmitted }}$ transmitted over the network considering minimum fragmentation.

$$
D a t a_{\text {transmitted }}=D a t a+\left\lceil\frac{\text { Data }}{M S D U_{\max }}\right\rceil \times L H
$$




$$
\begin{aligned}
L H & =\left\{\begin{array}{l}
\text { Headers } \quad \text { without acknowledgment } \\
\text { Headers }+t_{\mathrm{ACK}}+A C K \\
\text { with acknowledgment }
\end{array}\right. \\
\text { Headers } & =M A C_{\text {headers }}+P H Y_{\text {headers }}+\text { LIFS }
\end{aligned}
$$

Then we look for the slots needed by transmission of data payload of Data bytes in a GTS. The number $N$ of slots is provided by (4) and represented on figure 6.

$$
\mathrm{N}_{\text {slots }}=\left\lceil\frac{\text { Data }_{\text {transmitted }}}{\text { aBaseSlotDuration } \times 2^{S O}}\right\rceil
$$

Slots needed for a transmission can be evaluate and bring face to face with specification requirements (5).

$$
\sum_{i} \mathrm{~N}_{\text {slots }_{i}} \leq R_{\text {slots }}
$$

As an example, if we want to send 60 unacknowledged bytes on the network, we may select $S O=0, N_{\text {slots }}=$ 4 slots with (4) which satisfy the maximum $R_{\text {slots }}(2)$. But in the case data need to be acknowledged superframe order set to 0 induces $N_{\text {slots }}=5$ slots and it contradicts the maximum $R_{\text {slots }}=4$ slots obtained by (2). So in that case, a solution may be $S O=1$ which induces $\left(N_{\text {slots }}=3\right.$ slots $) \leq\left(R_{\text {slots }}=10\right.$ slots $)$.

\section{STUdy CASE}

Now we are able to set network parameters in order to satisfy the minimum requirements of such a network, we look for available real-time abilities based on an industrial case (fig. 1).

\section{A. Ability to poll sensors}

As a first evaluation, we study requirements needed for sensors transmissions. For that study, we consider a network set up with five industrial sensors transmitting and/or receiving data as described in Table I.

Table I

DATA EXCHANGES

\begin{tabular}{|c|c|c|c|}
\hline Device & Data send & Data received & Cycle \\
\hline $\mathrm{n}^{\circ} 1$ & 1 byte & - & $40 \mathrm{~ms}$ \\
\hline $\mathrm{n}^{\circ} 2$ & 8 bytes & - & $40 \mathrm{~ms}$ \\
\hline $\mathrm{n}^{\circ} 3$ & 4 bytes & - & $60 \mathrm{~ms}$ \\
\hline $\mathrm{n}^{\circ} 4$ & 8 bytes & 4 bytes & $60 \mathrm{~ms}$ \\
\hline $\mathrm{n}^{\circ} 5$ & 16 bytes & - & $100 \mathrm{~ms}$ \\
\hline
\end{tabular}

Even if sensors doesn't require the same period of cycle, we consider a cycle duration which allows any device to transmit during any superframe. Then the defined set of transmissions induce 6 GTS's; emission and reception implies for node 4 , the use of two different GTS's.

Such a configuration leads us to deduce the best network configuration corresponding to that usage. For that purpose, we compute $N_{i}$ for every node and we evaluate $C F P_{\text {slots }}=\Sigma_{i} N_{i}$. Evaluation of superframe order is given Table II and III.

Table II

SUPERFRAME ORDER EVALUATION, UNACKNOWLEDGMENT

\begin{tabular}{|c|c|c|c|c|}
\hline$S O$ & 0 & 1 & 2 & 3 \\
\hline$N_{1}$ & 2 & 1 & 1 & 1 \\
$N_{2}$ & 2 & 1 & 1 & 1 \\
$N_{3}$ & 2 & 1 & 1 & 1 \\
$N_{4}$ & 2 & 1 & 1 & 1 \\
$N_{4^{\prime}}$ & 2 & 1 & 1 & 1 \\
$N_{5}$ & 3 & 2 & 1 & 1 \\
\hline$C F P_{\text {slots }}$ & 13 & $\mathbf{7}$ & 6 & 6 \\
\hline$C F P_{\max }$ & 4 & $\mathbf{1 0}$ & 13 & 14 \\
\hline$S D(m s)$ & 15.36 & $\mathbf{3 0 . 7 2}$ & 61.44 & 122.88 \\
\hline
\end{tabular}

Table III

SUPERFRAME ORDER EVALUATION, ACKNOWLEDGMENT

\begin{tabular}{|c|c|c|c|c|}
\hline$S O$ & 0 & 1 & 2 & 3 \\
\hline$N_{1}$ & 3 & 2 & 1 & 1 \\
$N_{2}$ & 3 & 2 & 1 & 1 \\
$N_{3}$ & 3 & 2 & 1 & 1 \\
$N_{4}$ & 3 & 2 & 1 & 1 \\
$N_{4^{\prime}}$ & 3 & 2 & 1 & 1 \\
$N_{5}$ & 4 & 2 & 1 & 1 \\
\hline$C F P_{\text {slots }}$ & 19 & 12 & $\mathbf{6}$ & 6 \\
\hline$C F P_{\max }$ & 4 & 10 & $\mathbf{1 3}$ & 14 \\
\hline$S D(m s)$ & 15.36 & 30.72 & $\mathbf{6 1 . 4 4}$ & 122.88 \\
\hline
\end{tabular}

For that specific case, communications will be insured with the choice of $S O=2$ with acknowledgment whereas $S O=1$ is sufficient for unacknowledged communications. $S O=1$ corresponds to cycle duration equals $30.72 \mathrm{~ms}$ which satisfies all requirements (Cycle $\geq 40 \mathrm{~ms}$ ) whereas superframe duration of $61.44 \mathrm{~ms}$ doesn't. So we are able to satisfy sensors' communications in case we set up a network with $S O=1$ without acknowledgment.

\section{B. Data collection}

The previous analysis considers only communications from sensors to an unique receptor. If we want to allow end user to receive data from sensors, the coordinator should transfer those transmissions to the mobile device.

So, the coordinator will transmit every sensors transmission in one single GTS to the end user. Therefore, this communication includes the same number of frames. We 


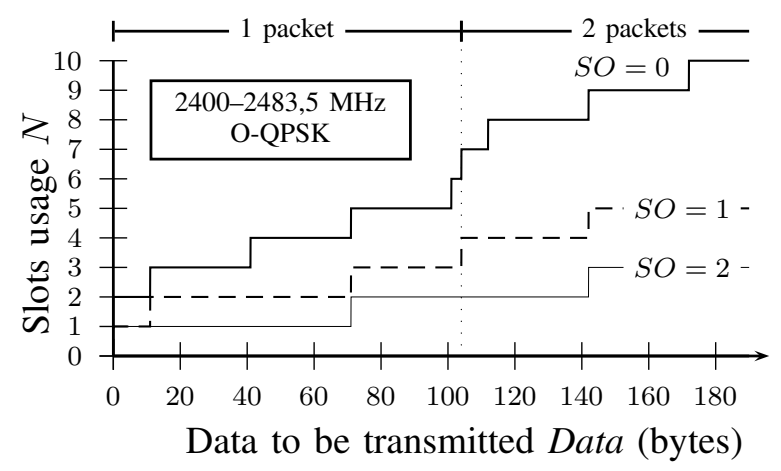

(a) unacknowledged

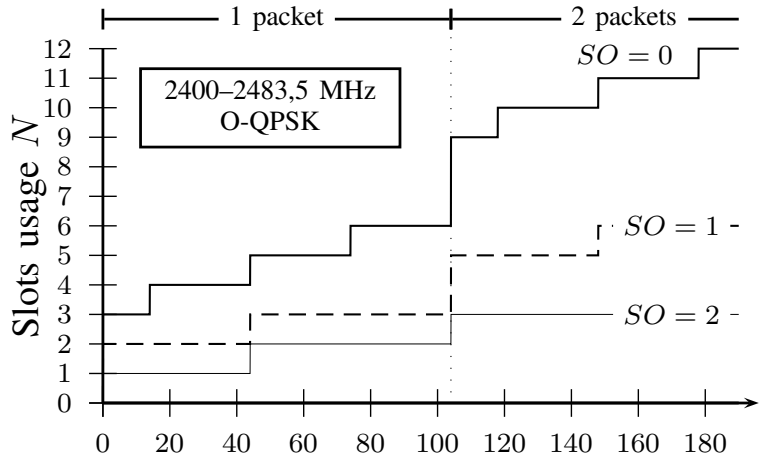

Data to be transmitted Data (bytes)

(b) acknowledged

Figure 6. Slots usage in relation with data transmission

must take into account the overhead of every frame and so we are unable to use (4) with Data $=\sum_{i=1}^{5}$ Data $_{i}$.

In order to calculate the number of slots required for transmission to maintenance hand-held, we need to compute $\operatorname{Data}_{\text {transmitted }_{6}}=\sum_{i=1}^{5} \operatorname{Data}_{\text {transmitted }_{i}}$ In our case, all frames are lower than $M P D U_{\max }$ (127 bytes), so we reduce the expression to Data $_{\text {transmitted }}=\sum_{i=1}^{5}$ Data $_{i}+5 \times$ $L H$. Then we deduct the superframe order corresponding to that network (Tables IV and V).

Table IV

SUPERFRAME ORDER EVALUATION, UNACKNOWLEDGMENT

\begin{tabular}{|c|c|c|c|c|}
\hline$S O$ & 0 & 1 & 2 & 3 \\
\hline$\Sigma N_{\text {slots }}$ & 11 & 11 & $\mathbf{8}$ & 7 \\
\hline$R_{\text {slots }}$ & 4 & 10 & $\mathbf{1 3}$ & 14 \\
\hline$S D(\mathrm{~ms})$ & 15.36 & 30.72 & $\mathbf{6 1 . 4 4}$ & 122.88 \\
\hline
\end{tabular}

Table V

SUPERFRAME ORDER EVALUATION, ACKNOWLEDGMENT

\begin{tabular}{|c|c|c|c|c|}
\hline$S O$ & 0 & 1 & 2 & 3 \\
\hline$\Sigma N_{\text {slots }}$ & 30 & 18 & $\mathbf{9}$ & 8 \\
\hline$R_{\text {slots }}$ & 4 & 10 & $\mathbf{1 3}$ & 14 \\
\hline$S D(m s)$ & 15.36 & 30.72 & $\mathbf{6 1 . 4 4}$ & 122.88 \\
\hline
\end{tabular}

In that case, superframe duration is $61.44 \mathrm{~ms}$ in either case and we can't satisfy the requirements of all sensors.

\section{METHODS COMPARISON}

GTS access method provides a simple polling mechanism for IEEE 802.15.4 devices. It may also be possible to implement a polling protocol through CSMA/CA.

In order to compare use of a CFP with CSMA/CA mechanisms, we consider such a polling protocol requiring an overhead of 1 byte. Coordinator sends poll request to a node and receives one or more poll response messages, depending on size message and needed fragmentation.
Comparison results are represented on figure 7 corresponding to a network with 5 nodes sending the same amount of data.

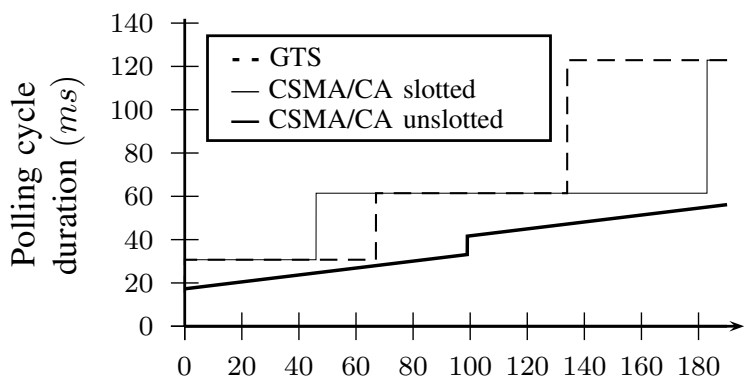

Data to be transmitted per station Dataresp (bytes)

(a) unacknowledged

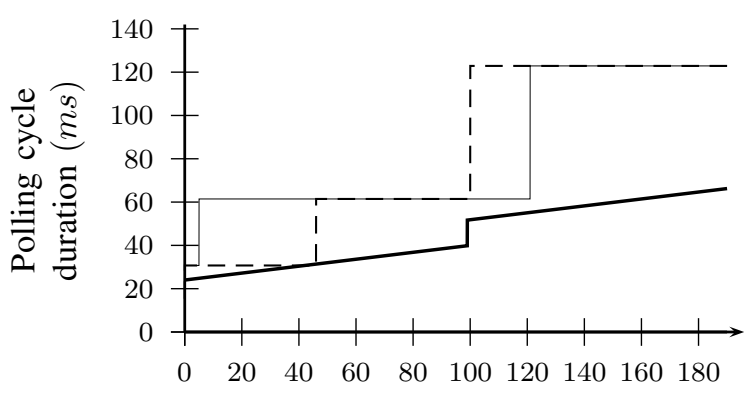

Data to be transmitted per station Dataresp (bytes)

(b) acknowledged

Figure 7. Access methods comparison w/ a 5 stations polling cycle

We observe that CSMA/CA unslotted provides lower cycle duration than GTS (except on very specific cases). But in what concerns CSMA/CA slotted, results are quite similar. GTS may provide lower cycle duration for low and average size messages whereas CSMA/CA is interesting for long messages, greater than 1 packet. 


\section{RELATIVE WORKS}

Our results focuses on a specific mechanism provided by IEEE 802.15.4 standard. Those results may be completed by [8], [9] who propose simulation results concerning CSMA/CA slotted.

Use of wireless networks for industrial applications may rely on the use of other standard, like IEEE 802.11 or IEEE 802.15.1.

IEEE 802.11 is currently most used for computer wireless networking. It relies on a CSMA/CA medium access which is not deterministic. A second medium access, named PCF (Point Coordination Function), exists and it allows to assure the transmission of a time constrained traffic. Some publications [10], [11] focus on IEEE 802.11 performances to support industrial constraints.

Bluetooth was developed in order to unbind equipments from their network wire. Due to some power limitation, its range of action is quite limited (mostly around $10 \mathrm{~m}$ ). Main applications of that protocol concern computer peripheral devices (printer, keyboard, cell phone ...). Transmission of real-time traffic over a Bluetooth network has been studied under multimedia conditions [12] as well as industrial cases [13]. Although that protocol is suitable for short range real-time communications, its intricate definition and high energy consumption matter with its use with small mobile devices.

\section{CONCLUSION}

That paper evaluates IEEE 802.15.4 temporal performances aimed to be used by industrial applications. It underlines some heavy limitations concerning cycle duration and number of devices that can communicate within Contention Free Period.

It reveals some difficulties to use IEEE 802.15.4 for heavy time constrained applications. Moreover main commercial Zigbee products, based on that standard, add more restrictions and it appears very rough to use such a technology for control command applications.

Use of IEEE 802.15.4 for more responsive use than supervision needs at least reconsideration of some standard values. It may also be possible to consider adaptation of some standard rules. Such propositions will be made in the latter based on simulation use cases. Simulation tools require some addition to fully support IEEE 802.15.4.

\section{ACKNOWLEDGMENT}

The authors gratefully acknowledge the support of the European Commission Sixth Framework programme for
Research and Technological Development. This paper summarises work performed as part of FP6 Integrated Project IP017498 DYNAMITE "Dynamic Decisions in Maintenance".

\section{REFERENCES}

[1] WINA, "The Wireless Industrial Network Alliance," http://www.wina.org, 2004-2007.

[2] A. Willig, "Redundancy concepts to increase transmission reliability in wireless industrial lans," in IEEE Transactions on Industrial Informatics, Aug. 2005.

[3] L. Krishnamurthy, R. Adler, P. Buonadonna, J. Chhabra, M. Flanigan, N. Kushalnagar, L. Nachman, and M. Yarvis, "Design and Deployment of Industrial Sensor Networks: Experience from a Semiconductor Plant and the North Sea," in 3rd international Conference on Embedded Networked Sensor Systems, 2005, pp. 64-75.

[4] H. Ramamurthy, B. Prabhu, R. Gadh, and A. Madni, "Wireless industrial monitoring and control using a smart sensor platform," in Sensors Journal. IEEE, May 2007, vol. 7, no. 5, pp. 611-618.

[5] DYNAMITE, "Dynamic Decisions in Maintenance," Europe, 2005-2009, FP6 Integrated Project IP017498.

[6] IEEE Computer Society, "IEEE Std 802.15.4-2006 (Revision of IEEE std 802.15.4-2003)," in IEEE Standard for Information technology-Telecommunications and information exchange between systems-Local and metropolitan area networks-Specific requirements - Part 15.4: Wireless Medium Access Control (MAC) and Physical Layer (PHY) Specifications for Low-Rate Wireless Personal Area Networks (LR-WPANs). IEEE, 8 Sep. 2006, pp. i-305.

[7] A. Koubâa, M. Alves, and E. Tovar, "Energy and delay tradeoff of the GTS allocation mechanism in IEEE 802.15.4 for wireless sensor networks," in Wiley Journal of Communication Systems, special issue on Energy-Efficient Network Protocols and Algorithms for Wireless Sensor Networks. John Wiley \& Sons, Ltd., 2006, pp. 1099-1131.

[8] A. Koubâa, M. Alves, B. Nefzi, and Y.-Q. Song, "Improving the IEEE 802.15.4 Slotted CSMA/CA MAC for Time-Critical Events in Wireless Sensor Networks," in Proc. of the Workshop of Real-Time Networks (RTN 2006), Jul. 2006.

[9] J. Mišić and C. J. Fung, "The impact of master-slave bridge access mode on the performance of multi-cluster 802.15.4 network," Computer Networks, vol. 51, pp. 2411-2449, 2007.

[10] G. Bianchi, "Performance analysis of the IEEE 802.11 distributed coordination function," IEEE Journal on Selected Areas in Communications, vol. 18, pp. 535-547, Mar. 2000.

[11] N. Krommenacker and V. Lecuire, "Building Industrial Communication Systems based on IEEE 802.11g wireless technology," in 10th IEEE International Conference on Emerging Tech. and Factory Automation, vol. 1, Sep. 2005, pp. 71-78.

[12] R. Kapoor, M. Kazantzidis, M. Gerla, and P. Johansson, "Multimedia support over bluetooth piconets," in Wireless Mobile Internet, ser. Proceedings of the first workshop on Wireless mobile internet, 2001, pp. 50-55.

[13] L. Lo Bello, M. Collotta, O. Mirabella, and T. Nolte, "Approaches to support real-time traffic over bluetooth networks," in Proceedings of the 4th international workshop on Real-Time Networks (RTN'05), J. Kaiser, Ed., Palma de Mallorca, Balearic Islands, Spain, 5 Jul. 2005, pp. 47-50. 